\title{
The finite-time ruin probability under the compound binomial risk model
}

\author{
Shuanming $\mathrm{Li}^{*}$ and Kristina P. Sendova ${ }^{\dagger}$
}

February 7, 2013

\begin{abstract}
We study the compound binomial ruin model, which is considered to be the discrete analogue of the classical compound Poisson model. Our key result is a simple approach for inverting a generating function whose argument is the discount factor when we know the inverse of the same generating function, which this time has argument that is the solution to Lundberg's equation. The main idea comes from a result in Dickson and Willmot (2005) who discuss the classical model. We are then able to derive the probability distribution of the time to ruin and to go beyond the results in Dickson and Willmot (2005) by deducing the distribution of the first hitting time of a specific level and the duration of the time when the surplus is negative. The paper contains several illustrative examples where specific claim-amount distributions are considered.
\end{abstract}

Keywords: Compound binomial risk model; Duration of negative surplus; Generalized Lundberg's equation; Generating function; Finite time ruin probability; First hitting time.

\section{Introduction}

The compound binomial model, first proposed by Gerber (1988), is a discrete analog of the classical compound Poisson model in risk theory. It is a fully discrete-time model where premiums, claim amounts, and the initial surplus are assumed to be integer valued, but may be used as an approximation to the continuous-time compound Poisson model. Gerber (1988) and Shiu (1989) considered the

\footnotetext{
${ }^{*}$ Centre for Actuarial Studies, Department of Economics, The University of Melbourne, Victoria 3010, Australia

$\dagger$ (corresponding author) Department of Statistical and Actuarial Sciences, The University of Western Ontario, 1151 Richmond Street North, London, Ontario, N6A 5B7, Canada, e-mail: ksendova@stats.uwo.ca
} 
ultimate ruin probability for the model, while Willmot (1993) studied finite-time ruin probabilities. For other references on related topics see, for example, Dickson (1994), Li and Garrido (2002), and Pavlova and Willmot (2004). Several extensions to the compound binomial risk model may be found in Cossette et al. (2003, 2004a), Landriault (2008), Li (2005a,b), Yang et al. (2009), Yuen and Guo (2001, 2006), and references therein.

Unlike continuous-time risk models, discrete-time risk models have not attracted as much attention and the literature counts fewer contributions. Yet, discrete-time risk models also have their special features and are closer to reality. Results under such settings may be given a simpler interpretation than their analogue in the continuous-time setting. Such results are also of independent interest since formulas for discrete-time models are of a recursive nature and readily programmable in practice, while still reproducing the continuous analogue results as limiting cases. It is well known that explicit expressions for some ruin-related quantities do not exist in continuous-time risk models with heavy-tailed claims. Instead, results for the discrete-time risk models can be used as approximations or bounds for the corresponding results in continuous time, see Dickson et al. (1995) and Cossette et al. (2004b) for the approximating procedures.

In this paper, special consideration is given to the probability of ruin within a finite time interval. This type of probabilities prove to be more difficult to tackle in general than the widely studied probability of ultimate ruin. As it is well known, the latter probability may be obtained from the former.

We now proceed with the description of the compound binomial model. Assume that the premium income for each period is one and the number of claims up to time (or period) $n \in \mathbb{N}:=\{0,1,2, \ldots\}$ is governed by a binomial process $\{N(n) ; n \in \mathbb{N}\}$ with

$$
N(n)=I_{1}+I_{2}+\cdots+I_{n}, \quad n \in \mathbb{N}^{+}:=\{1,2,3, \ldots\}
$$

with $N(0)=0$, where $I_{1}, I_{2}, \ldots$, are independent and identically distributed (i.i.d.) Bernoulli random variables with mean $q \in(0,1)$. That is, in any time period there is at most one claim; the probability of having a claim is $q$ and the probability of no claim is $1-q$. The occurrence of the claims in different time periods are assumed to be independent events.

More formally, let $W_{1}$ denote the time until the first claim and let $W_{n}$ denote the time between the $(n-1)$ st and $n$th claims for $n=2,3,4, \ldots$ Let $\left\{W_{i}\right\}_{i=1}^{\infty}$ be a sequence of i.i.d. random variables, with discrete probability function (p.f.) $\ell(t), t \in \mathbb{N}^{+}$.

Define by $\tau_{1}=W_{1}$ the occurrence time of the first claim and by $\tau_{k}=\sum_{i=1}^{k} W_{i}$ the occurrence time of the $k$-th claim. Then $N(n)$ defined by (1.1) may also be written as

$$
N(n)=\max \left\{k: W_{1}+W_{2}+\cdots+W_{k} \leq n\right\}=\max \left\{k: \tau_{k} \leq n\right\}, \quad n \in \mathbb{N}^{+}
$$


When each $W_{i}$ follows a zero-truncated geometric distribution, i.e., its p.f. is

$$
\ell(t)=q(1-q)^{t-1}, \quad t \in \mathbb{N}^{+}, q \in(0,1),
$$

then $\{N(n) ; n \in \mathbb{N}\}$ is called a binomial process with

$$
\mathbb{P}\{N(n)=k\}=\left(\begin{array}{l}
n \\
k
\end{array}\right) q^{k}(1-q)^{n-k}, \quad k=0,1, \ldots, n .
$$

The claim amounts $\left\{X_{i}\right\}_{i=1}^{\infty}$ are mutually independent, identically distributed, positive, integervalued, random variables with common p.f. $p(x)=\mathbb{P}\{X=x\}, x \in \mathbb{N}^{+}$, cumulative distribution function (c.d.f.) $P(y)=\sum_{x=1}^{y} p(x)$, probability generating function (p.g.f.) $\hat{p}(z)=\sum_{x=1}^{\infty} p(x) z^{x}$ and $k$ th moment denoted by $\mu_{k}=\sum_{x=1}^{\infty} x^{k} p(x)$. The claim sizes are also independent of the counting process $\{N(n) ; n \in \mathbb{N}\}$. Then, the surplus of an insurance company at time $n$ is described as

$$
U(n)=u+n-\sum_{i=1}^{N(n)} X_{i}, \quad n \in \mathbb{N}^{+},
$$

where $U(0)=u \in \mathbb{N}$ is the initial surplus. We further assume that $q \mu_{1}<1$, providing a positive loading condition. This is the so-called discrete-time compound binomial risk model first proposed by Gerber (1988). The model defined in (1.2) may be rewritten as

$$
U(n)=u+n-\sum_{i=1}^{n} Y_{i}, \quad n \in \mathbb{N}^{+},
$$

where $Y_{i}=I_{i} X_{i}$ is the claim amount in period $i$, with p.f.

$$
b(x)=\left\{\begin{array}{ll}
1-q, & x=0 \\
q p(x), & x \in \mathbb{N}^{+}
\end{array},\right.
$$

c.d.f. $B(x)=1-\bar{B}(x)=\sum_{y=0}^{x} b(y)$, p.g.f. $\hat{b}(z)=\sum_{x=0}^{\infty} z^{x} b(x)=1-q+q \hat{p}(z)$, and $k$ th moment $q \mu_{k}$. Note that $b(x)$ is a zero-modified version of $p(x)$.

We define the random variable $T=\min \left\{t \in \mathbb{N}^{+} ; U(t)<0\right\}$ to be the time of ruin and

$$
\psi(u)=\mathbb{P}\{T<\infty \mid U(0)=u\}, \quad u \in \mathbb{N},
$$

to be the ultimate ruin probability (also known as the eventual ruin probability or the infinite-time ruin probability). Although the above definition of $T$ yields simpler expressions for the quantities of actuarial interest, it differs from the definition in Cheng et al. (2000) and Li and Garrido (2002) so some adjustments need to be made when we refer to those papers. 
Define further

$$
w(u ; n)=\mathbb{P}\{T=n \mid U(0)=u\}, \quad u \in \mathbb{N}, n \in \mathbb{N}^{+},
$$

to be the probability function of $T$. We have then the following important relationships

$$
\psi(u)=\sum_{n=1}^{\infty} w(u ; n), \quad u \in \mathbb{N},
$$

and

$$
\psi(u ; n)=\mathbb{P}\{T \leq n \mid U(0)=u\}=\sum_{j=1}^{n} w(u ; j), \quad u \in \mathbb{N}, n \in \mathbb{N}^{+} .
$$

For $v \in(0,1]$, define

$$
\phi_{T}(u)=\mathbb{E}\left\{v^{T} I(T<\infty) \mid U(0)=u\right\}, \quad u \in \mathbb{N} .
$$

to be the (defective) p.g.f. of $T$.

The associated generalized Lundberg's equation plays an important role in what follows. In this relation, we recall Lemma $1 \mathrm{in} \mathrm{Li}$ and Garrido (2002).

Lemma 1.1. The generalized Lundberg's fundamental equation for the compound binomial risk model (1.3) is

$$
\frac{\hat{b}(z)}{z}=\frac{1}{v}, \quad z>0,
$$

and has one positive solution $\rho=\rho(v) \in(0,1)$. If there exists $\kappa>1$ such that $\hat{b}(z)<\infty$, for all $z<\kappa$ and $\lim _{z \rightarrow \kappa-} \hat{b}(z)=\infty$, it has another solution $R=R(v) \in(1, \infty)$.

Moreover,

1. $\rho(v) \in(0, v)$;

2. $\lim _{v \rightarrow 0} \rho(v)=0$ and $\lim _{v \rightarrow 1} \rho(v)=1$;

3. $\rho^{\prime}(v)=\frac{\hat{b}(\rho)}{1-v \hat{b}^{\prime}(\rho)}$, with $\lim _{v \rightarrow 0} \rho^{\prime}(v)=b(0)$ and $\lim _{v \rightarrow 1} \rho^{\prime}(v)=\frac{1}{1-q \mu_{1}}$.

To conclude this section, we describe how the paper is organized. Section 2 contains a technical result connected to the generating functions. Subsequently, Section 3 discusses the distribution of the time until the first crossing of a pre-specified level, starting with no initial surplus. Section 4 is concerned with the distribution of the time to ruin. Lastly, in Section 5 we consider the possibility of a recovery after ruin by allowing the surplus to remain negative. We investigate the length of time that elapses until recovery. Examples are provided throughout the paper to illustrate our results. 


\section{A generating function transform relationship}

The purpose of this section is to formally state a theoretical result that is useful for determining the relationship between one and the same generating function interpreted either with respect to argument $\rho$ or $v$.

It follows from the Lagrange's implicit function theorem (see, for example, Goulden and Jackson, 1983) that if $y$ is determined by the following implicit function

$$
y=a+x h(y)
$$

then for any analytic function $\eta$, we have

$$
\eta(y)=\eta(a)+\left.\sum_{n=1}^{\infty} \frac{x^{n}}{n !} \frac{d^{n-1}}{d z^{n-1}}\left[\eta^{\prime}(z) h^{n}(z)\right]\right|_{z=a},
$$

and in particular,

$$
y=a+\left.\sum_{n=1}^{\infty} \frac{x^{n}}{n !} \frac{d^{n-1}}{d z^{n-1}} h^{n}(z)\right|_{z=a} .
$$

For our compound binomial risk model, $\rho$ satisfies $\rho=v \hat{b}(\rho)$ by Lemma 1.1. Therefore,

$$
\eta(\rho)=\eta(0)+\left.\sum_{n=1}^{\infty} \frac{v^{n}}{n !} \frac{d^{n-1}}{d z^{n-1}}\left[\eta^{\prime}(z) \sum_{x=0}^{\infty} z^{x} b^{n *}(x)\right]\right|_{z=0}
$$

where $b^{n *}$ is the $n$-fold convolution of $b$ with itself. In particular, $\rho$ may be computed explicitly by

$$
\begin{aligned}
\rho & =\left.\sum_{n=1}^{\infty} \frac{v^{n}}{n !} \frac{d^{n-1}}{d z^{n-1}}\left[\sum_{x=0}^{\infty} z^{x} b^{n *}(x)\right]\right|_{z=0} \\
& =\left.\sum_{n=1}^{\infty} \frac{v^{n}}{n !} \sum_{x=n-1}^{\infty} x^{(n-1)} z^{x-(n-1)} b^{n *}(x)\right|_{z=0} \\
& =\sum_{n=1}^{\infty} \frac{v^{n}}{n} b^{n *}(n-1)
\end{aligned}
$$

where $x^{(n)}=x(x-1)(x-2) \cdots(x-n+1)$ is the $n$th factorial power of $x$. Furthermore, we have the following key result.

Lemma 2.1. For an arbitrary function $f(y), y \in \mathbb{N}^{+}$, such that

$$
\hat{f}(\rho)=\sum_{y=1}^{\infty} \rho^{y} f(y)=\sum_{n=1}^{\infty} v^{n} h(n)=\hat{h}(v),
$$


with $\rho=\rho(v)$ as defined by Lemma 1.1, we have

$$
h(n)=\frac{1}{n} \sum_{y=1}^{n} y f(y) b^{n *}(n-y), \quad n \in \mathbb{N}^{+} .
$$

Proof. Formula (2.1) with $\eta(\rho)=\rho^{y}, y \in \mathbb{N}^{+}$, yields

$$
\begin{aligned}
\rho^{y} & =\left.\sum_{n=1}^{\infty} \frac{v^{n}}{n !} \frac{d^{n-1}}{d z^{n-1}}\left[y z^{y-1} \sum_{x=0}^{\infty} z^{x} b^{n *}(x)\right]\right|_{z=0} \\
& =\left.y \sum_{n=1}^{\infty} \frac{v^{n}}{n !} \frac{d^{n-1}}{d z^{n-1}}\left[\sum_{x=0}^{\infty} z^{x+y-1} b^{n *}(x)\right]\right|_{z=0} \\
& =\left.y \sum_{n=1}^{\infty} \frac{v^{n}}{n !} \sum_{x=n-y}^{\infty}(x+y-1)^{(n-1)} z^{x+y-n} b^{n *}(x)\right|_{z=0} \\
& =y \sum_{n=1}^{\infty} \frac{v^{n}}{n} b^{n *}(n-y)=y \sum_{n=y}^{\infty} \frac{v^{n}}{n} b^{n *}(n-y) .
\end{aligned}
$$

Thus, for a function $f(y), y \in \mathbb{N}^{+}$, satisfying (2.2), we have

$$
\begin{aligned}
\hat{f}(\rho) & =\sum_{y=1}^{\infty} \rho^{y} f(y)=\sum_{y=1}^{\infty} y f(y) \sum_{n=y}^{\infty} \frac{v^{n}}{n} b^{n *}(n-y) \\
& =\sum_{n=1}^{\infty} \frac{v^{n}}{n} \sum_{y=1}^{n} y f(y) b^{n *}(n-y)=\sum_{n=1}^{\infty} v^{n} h(n)=\hat{h}(v),
\end{aligned}
$$

with $h(n)$ defined by (2.3) as required.

Remark 2.1. $\quad$ 1. The $n$-fold convolution $b^{n *}$ may be calculated by the following recursive formula

$$
b^{n *}(x)=\frac{1}{b(0)} \sum_{j=1}^{x}\left[(n+1) \frac{j}{x}-1\right] b(j) b^{n *}(x-j), \quad x, n \in \mathbb{N}^{+},
$$

where the starting value is $b^{n *}(0)=[b(0)]^{n}$, see Dickson and Willmot (2005), p. 22 .

2. As $[\hat{b}(z)]^{n}=[1-q+q \hat{p}(z)]^{n}=\sum_{k=0}^{n}\left(\begin{array}{l}n \\ k\end{array}\right) q^{k}(1-q)^{n-k}[\hat{p}(z)]^{k}$, we have

$$
b^{n *}(x)=\sum_{k=0}^{n}\left(\begin{array}{l}
n \\
k
\end{array}\right) q^{k}(1-q)^{n-k} p^{k *}(x)=\sum_{k=0}^{n \wedge x}\left(\begin{array}{l}
n \\
k
\end{array}\right) q^{k}(1-q)^{n-k} p^{k *}(x),
$$

where $p^{0 *}(x)=I(x=0)$ and $n \wedge x=\min (n, x)$. Thus $h(n)$ may be re-expressed as

$$
h(n)=\frac{1}{n} \sum_{k=0}^{n}\left(\begin{array}{l}
n \\
k
\end{array}\right) q^{k}(1-q)^{n-k} \sum_{y=1}^{n} y f(y) p^{k *}(n-y), \quad n \in \mathbb{N}^{+},
$$




$$
=(1-q)^{n} f(n)+\frac{1}{n} \sum_{k=1}^{n}\left(\begin{array}{l}
n \\
k
\end{array}\right) q^{k}(1-q)^{n-k} \sum_{y=1}^{n} y f(y) p^{k *}(n-y) .
$$

3. Equation (2.6) is the discrete analogue of equation (4) in Dickson (2005) for the classical compound Poisson risk model.

\section{The distribution of the first hitting time}

The first application of the above results appears in this section where we consider the time $T_{x}$ that elapses until the surplus process $U$ achieves a pre-specified level $x$. We may assume without loss of generality that the initial surplus equals zero.

More precisely, define

$$
T_{x}:=\inf \{n>0: U(n)=x, U(0)=0\}, \quad x \in \mathbb{N}^{+},
$$

to be the first time that the surplus process arrives at level $x$ from an initial surplus of 0 .

Proposition 3.1. The probability function of the hitting time $T_{x}$ is

$$
f(x, n)=\mathbb{P}\left\{T_{x}=n\right\}=\frac{x}{n} b^{n *}(n-x), \quad n \geq x .
$$

Proof. Let $R_{v}(x)=\mathbb{E}\left\{v^{T_{x}}\right\}, v \in(0,1]$, be the p.g.f. of $T_{x}$. Cheng et al. (2000) show that $R_{v}(x)=\rho^{x}$, where $\rho \in(0,1)$ is the solution to Lundberg's equation (1.5) (see identity (3.7) in Cheng et al., 2000).

It now follows from equation (2.4) that

$$
R_{v}(x)=\mathbb{E}\left\{v^{T_{x}}\right\}=\sum_{n=x}^{\infty} v^{n} f(x, n)=\rho^{x}=x \sum_{n=x}^{\infty} \frac{v^{n}}{n} b^{n *}(n-x),
$$

which yields the desired result.

In particular, Proposition 3.1 implies that $\mathbb{P}\left\{T_{x}=x\right\}=f(x, x)=b^{x *}(0)=[b(0)]^{x}=(1-q)^{x}$. That is to say, the surplus arrives at level $x$ at time $x$ if there are no claims in the first $x$ periods, and the probability of this event is $(1-q)^{x}$.

Example 3.1. (Geometric claims) If $p(x)=(1-\theta) \theta^{x-1}, x \in \mathbb{N}^{+}, \theta \in(0,1)$, we have

$$
p^{k *}(x)=\left(\begin{array}{l}
x-1 \\
x-k
\end{array}\right)(1-\theta)^{k} \theta^{x-k}, \quad x \geq k,
$$


and for $n \in \mathbb{N}^{+}$,

$$
b^{n *}(x)= \begin{cases}(1-q)^{n}, & x=0 \\
\sum_{k=1}^{n \wedge x}\left(\begin{array}{l}
n \\
k
\end{array}\right)\left(\begin{array}{l}
x-1 \\
x-k
\end{array}\right) q^{k}(1-q)^{n-k}(1-\theta)^{k} \theta^{x-k}, & x \in \mathbb{N}^{+}\end{cases}
$$

then

$$
f(x, n)=\frac{x}{n} b^{n *}(n-x)= \begin{cases}(1-q)^{n} & n=x \\
\frac{x}{n} \theta^{-x} \sum_{k=1}^{n-x}\left(\begin{array}{l}
n \\
k
\end{array}\right)\left(\begin{array}{ll}
n-x-1 \\
n-x-k
\end{array}\right)[q(1-\theta)]^{k}[\theta(1-q)]^{n-k}, & n>x\end{cases}
$$

In particular, if $\theta=q$, i.e., $b(x)=(1-q) q^{x}, x \in \mathbb{N}$, then

$$
\begin{aligned}
f(x, n) & = \begin{cases}(1-q)^{n}, & n=x \\
\frac{x}{n}(1-q)^{n} q^{n-x} \sum_{k=1}^{n-x}\left(\begin{array}{l}
n \\
k
\end{array}\right)\left(\begin{array}{c}
n-x-1 \\
n-x-k
\end{array}\right), & n>x\end{cases} \\
& =\frac{x}{n}\left(\begin{array}{c}
2 n-x-1 \\
n-x
\end{array}\right)(1-q)^{n} q^{n-x}, \quad n \geq x .
\end{aligned}
$$

Example 3.2. (Discrete phase-type claims) In this example, we assume that $b(x)$ is a discrete phasetype distribution with representation $(\overrightarrow{\boldsymbol{\alpha}}, \mathbf{T})$, where $\overrightarrow{\boldsymbol{\alpha}}=\left(\alpha_{1}, \ldots, \alpha_{m}\right)$ with $\sum_{i=1}^{m} \alpha_{i}=q$. Then $b(x)$ is then given by

$$
\begin{aligned}
& b(0)=1-q, \\
& b(x)=\overrightarrow{\boldsymbol{\alpha}} \mathbf{T}^{x-1} \overrightarrow{\mathbf{t}}^{\top}, \quad x \in \mathbb{N}^{+},
\end{aligned}
$$

where $\mathbf{T}=\left(t_{i, j}\right)_{m \times m}$ is a substochastic matrix and $\overrightarrow{\mathbf{t}}^{\top}=\left(t_{1,0}, t_{2,0}, \ldots, t_{m, 0}\right)^{\top}=(\mathbf{I}-\mathbf{T}) \overrightarrow{\mathbf{1}}^{\top}$ with $\mathbf{I}$ being the $m \times m$ identity matrix and $\overrightarrow{\mathbf{1}}=(1,1, \ldots, 1)$ being a $1 \times m$ row vector. It follows from Latoche and Ramaswami (1999) that the $n$-fold convolution of a phase-type distribution with representation $(\boldsymbol{\alpha}$, $\mathbf{T})$ is still a phase-type distribution with representation $(\overrightarrow{\boldsymbol{\alpha}}(n), \boldsymbol{\mathcal { T }}(n))$, where $\overrightarrow{\boldsymbol{\alpha}}(n)=(\overrightarrow{\boldsymbol{\alpha}}, \overrightarrow{\mathbf{0}}, \ldots, \overrightarrow{\mathbf{0}})$ is a $1 \times m n$ row vector,

$$
\boldsymbol{\mathcal { T }}(n)=\left[\begin{array}{cccccc}
\mathbf{T} & \overrightarrow{\mathbf{t}}^{\top} \overrightarrow{\boldsymbol{\alpha}} & \mathbf{0}(n) & \cdots & \mathbf{0}(n) & \mathbf{0}(n) \\
\mathbf{0}(n) & \mathbf{T} & \overrightarrow{\mathbf{t}}^{\top} \overrightarrow{\boldsymbol{\alpha}} & \cdots & \mathbf{0}(n) & \mathbf{0}(n) \\
\vdots & \vdots & \vdots & \cdots & \vdots & \vdots \\
\mathbf{0}(n) & \mathbf{0}(n) & \mathbf{0}(n) & \cdots & \mathbf{T} & \overrightarrow{\mathbf{t}}^{\top} \overrightarrow{\boldsymbol{\alpha}} \\
\mathbf{0}(n) & \mathbf{0}(n) & \mathbf{0}(n) & \cdots & \mathbf{0}(n) & \mathbf{T}
\end{array}\right]
$$


and $\mathbf{0}(n)=(0)_{m \times m}$. Further, the corresponding absorption rates vector is denoted by $\overrightarrow{\mathbf{t}}(n)=$ $\left(\overrightarrow{\mathbf{0}}^{\top}, \overrightarrow{\mathbf{0}}^{\top}, \ldots, \overrightarrow{\mathbf{0}}^{\top}, \overrightarrow{\mathbf{t}}^{\top}\right)^{\top}$ which is a $m n \times 1$ column vector. Then

$$
\begin{aligned}
& b^{n *(0)}=[b(0)]^{n}=(1-q)^{n}, \\
& b^{n *}(x)=\overrightarrow{\boldsymbol{\alpha}}(n) \mathcal{T}^{x-1}(n) \overrightarrow{\mathbf{t}}^{\top}(n), \quad x \in \mathbb{N}^{+} .
\end{aligned}
$$

and

$$
f(x, n)=\frac{x}{n} b^{n *}(n-x)=\left\{\begin{array}{ll}
(1-q)^{n}, & n=x \\
\frac{x}{n} \overrightarrow{\boldsymbol{\alpha}}(n) \boldsymbol{\mathcal { T }}^{n-x-1}(n) \overrightarrow{\mathbf{t}}^{\top}(n), & n>x
\end{array} .\right.
$$

Remark 3.1. It follows from Latoche and Ramaswami (1999) that every probability function with finite support on the nonnegative integers is of discrete phase-type. Let probability function $b(x)$ be defined on $\{0,1, \ldots, m\}$. It is easy to show that $\{b(x)\}_{x=0}^{m}$ is a phase-type distribution with representation $(\overrightarrow{\boldsymbol{\alpha}}, \mathbf{T})$, where $\overrightarrow{\boldsymbol{\alpha}}=(b(1), b(2), \ldots, b(m))$ and $\mathbf{T}=(I(i=j+1))_{i, j=1}^{m}$ with $I(i=$ $j+1)=1$, if $i=j+1$, and 0 , otherwise. Therefore we may employ (3.3) to calculate the probability function of $T_{x}$. Moreover, if $m$ is small, calculating $p^{n *}(x)$ using (3.3) is time efficient. If $m$ is very large, we need to deal with a $m n \times m n$ matrix $\mathcal{T}(n)$, in particular, if $n$ is also larger, the computation of $p^{n *}(x)$ is much slower than when the recursive formula (2.5) is eployed.

\section{The probability function of the time to ruin}

In this section, we aim to find the probability function of the time to ruin by inverting its probability generating function, which has been studied as a special case of the Gerber-Shiu function in Cheng et al. (2000) and Li and Garrido (2002). Although our approach is inspired by Dickson and Willmot (2005), there are substantial differences in the calculation techniques that are required.

\subsection{The probability function of $T$ when $u=0$}

We begin by considering the case of no initial surplus. Although it is of interest on its own, an expression of $\phi_{T}(0)$ is required for determining the p.f. of $T$ when the initial surplus is non-zero.

Lemma 4.1. When $n \in \mathbb{N}^{+}$, the p.f. of the time of ruin is provided by

$$
w(0 ; n)=\frac{1}{1-q}\left[\frac{1}{n} \sum_{y=1}^{n} y b^{n *}(n-y)-\frac{1}{n+1} \sum_{y=1}^{n+1} y b^{(n+1) *}(n+1-y)\right] \text {. }
$$


Proof. We begin by implementing the last equation on p. 6 in Li and Garrido (2002) with the penalty function being identically equal to 1 .

$$
\begin{aligned}
\phi_{T}(0) & =\mathbb{E}\left\{v^{T} I(T<\infty) \mid U(0)=0\right\} \\
& =\frac{\rho}{b(0)} \sum_{x=0}^{\infty} \sum_{y=1}^{\infty} \rho^{x} b(x+y+1)=\frac{\rho}{1-q} \sum_{x=0}^{\infty} \sum_{y=x+2}^{\infty} \rho^{x} b(y) \\
& =\frac{\rho}{1-q} \sum_{y=2}^{\infty} \sum_{x=0}^{y-2} \rho^{x} b(y)=\frac{\rho}{1-q} \sum_{y=2}^{\infty} b(y) \frac{1-\rho^{y-1}}{1-\rho} \\
& =1-\frac{\hat{b}(\rho)-\rho}{(1-q)(1-\rho)}=1-\frac{\rho(1 / v-1)}{(1-q)(1-\rho)}=1-\frac{1}{1-q}\left(\frac{1}{v}-1\right) \sum_{y=1}^{\infty} \rho^{y} .
\end{aligned}
$$

Substituting (2.4) into (4.3), we have

$$
\begin{aligned}
\phi_{T}(0) & =1-\frac{1}{1-q}\left(\frac{1}{v}-1\right) \sum_{y=1}^{\infty} y \sum_{n=y}^{\infty} \frac{v^{n}}{n} b^{n *}(n-y) \\
& =1-\frac{1}{1-q}\left(\frac{1}{v}-1\right) \sum_{n=1}^{\infty} \frac{v^{n}}{n} \sum_{y=1}^{n} y b^{n *}(n-y) \\
& =\frac{1}{1-q} \sum_{n=1}^{\infty} \frac{v^{n}}{n} \sum_{y=1}^{n} y b^{n *}(n-y)-\frac{1}{1-q} \sum_{n=2}^{\infty} \frac{v^{n-1}}{n} \sum_{y=1}^{n} y b^{n *}(n-y) \\
& =\frac{1}{1-q} \sum_{n=1}^{\infty} v^{n}\left[\frac{1}{n} \sum_{y=1}^{n} y b^{n *}(n-y)-\frac{1}{n+1} \sum_{y=1}^{n+1} y b^{(n+1) *}(n+1-y)\right] .
\end{aligned}
$$

Taking into account that

$$
\phi_{T}(0)=\mathbb{E}\left\{v^{T} I(T<\infty) \mid U(0)=0\right\}=\sum_{n=1}^{\infty} w(0 ; n) v^{n}
$$

we deduce the desired result.

Lemma 4.2. The probability generating function of the time to ruin with zero initial surplus may be expressed as

$$
\phi_{T}(0)=\frac{q \mu_{1}}{1-q} \sum_{x=1}^{\infty} \rho^{x} b_{1}(x)
$$

where $b_{1}(x)=\bar{B}(x) /\left(q \mu_{1}\right), x \in \mathbb{N}$, is the equilibrium p.f. of $b$, with $q \mu_{1}=\sum_{x=1}^{\infty} x b(x)$ being the mean of the claim amounts.

Proof. Identity (4.2) is equivalent to

$$
\phi_{T}(0)=\mathbb{E}\left\{v^{T} I(T<\infty) \mid U(0)=0\right\}=\frac{1}{1-q} \sum_{x=0}^{\infty} \rho^{x} \sum_{y=x+2}^{\infty} b(y)=\frac{1}{1-q} \sum_{x=0}^{\infty} \rho^{x+1} \bar{B}(x+1),
$$


which leads to the desired result.

There is an alternative way to obtain $w(0, n)$. Namely, substituting (2.4) into (4.4) and changing the order of summation, we have

$$
\phi_{T}(0)=\frac{q \mu_{1}}{1-q} \sum_{n=1}^{\infty} v^{n} \sum_{y=1}^{n} \frac{y b_{1}(y) b^{n *}(n-y)}{n} .
$$

Inversion yields another expression for $w(0 ; n)$, which is

$$
w(0 ; n)=\frac{q \mu_{1}}{1-q} \sum_{y=1}^{n} \frac{y b_{1}(y) b^{n *}(n-y)}{n}, \quad n \in \mathbb{N}^{+} .
$$

\subsection{The probability function of $T$ when $u>0$}

We turn our attention now to finding an explicit expression for $w(u, n)$ when $u \in \mathbb{N}^{+}$. We include first two simple examples to illustrate the complexity of the finite-time ruin probability.

Example 4.1. (Geometric claims) If $p(x)=(1-\theta) \theta^{x-1}, x \in \mathbb{N}^{+}, \theta \in(0,1)$, then it follows from Li (2005b) that

$$
\phi_{T}(u)=\frac{1}{1-\theta} R^{-(u+1)}-\frac{\theta}{1-\theta} R^{-u}, \quad u \in \mathbb{N}^{+},
$$

where $R>1$ is the solution to

$$
z=v \hat{b}(z)=v[1-q+q \hat{p}(z)]=v\left[1-q+q \frac{(1-\theta) z}{1-\theta z}\right] .
$$

Solving for $z$ yields

$$
\begin{aligned}
& R=\frac{1-v[q(1-\theta)-\theta(1-q)]+\sqrt{\{1-v[q(1-\theta)-\theta(1-q)]\}^{2}-4 v(1-q) \theta}}{2 \theta}, \\
& \rho=\frac{1-v[q(1-\theta)-\theta(1-q)]-\sqrt{\{1-v[q(1-\theta)-\theta(1-q)]\}^{2}-4 v(1-q) \theta}}{2 \theta} .
\end{aligned}
$$

Multiplying these two expressions produces $R=v(1-q) /(\theta \rho)$. Equation (4.6) may be re-written as

$$
\phi_{T}(u)=\frac{1}{1-\theta}\left(\frac{\theta}{1-q}\right)^{u+1}\left(\frac{\rho}{v}\right)^{u+1}-\frac{\theta}{1-\theta}\left(\frac{\theta}{1-q}\right)^{u}\left(\frac{\rho}{v}\right)^{u} .
$$

Note that the ruin probability $\psi(u)$ may be deduced as a limiting case, i.e.,

$$
\psi(u)=\lim _{v \rightarrow 1-} \phi_{T}(u)=\frac{q}{1-\theta}\left(\frac{\theta}{1-q}\right)^{u+1}, \quad u \in \mathbb{N} .
$$


It follows from (2.4) that

$$
\left(\frac{\rho}{v}\right)^{u}=u \sum_{k=0}^{\infty} \frac{v^{k}}{u+k} b^{(u+k) *}(k) .
$$

Inverting (4.7) and applying (3.2) produce

$$
\begin{aligned}
w(u ; n)= & \frac{u+1}{1-\theta}\left(\frac{\theta}{1-q}\right)^{u+1} \frac{b^{(u+1+n) *}(n)}{u+1+n}-\frac{\theta u}{1-\theta}\left(\frac{\theta}{1-q}\right)^{u} \frac{b^{(u+n) *}(n)}{u+n} \\
= & \frac{\theta^{u+1}}{1-\theta} \frac{u+1}{n+u+1} \sum_{k=1}^{n}\left(\begin{array}{c}
n+u+1 \\
k
\end{array}\right)\left(\begin{array}{c}
n-1 \\
n-k
\end{array}\right)[q(1-\theta)]^{k}[\theta(1-q)]^{n-k} \\
& -\frac{\theta^{u+1}}{1-\theta} \frac{u}{n+u} \sum_{k=1}^{n}\left(\begin{array}{c}
n+u \\
k
\end{array}\right)\left(\begin{array}{c}
n-1 \\
n-k
\end{array}\right)[q(1-\theta)]^{k}[\theta(1-q)]^{n-k}
\end{aligned}
$$

In particular, if $\theta=q$, i.e., $b(x)=q(1-q)^{x}, x \in \mathbb{N}$, we have for $u \in \mathbb{N}$,

$$
\begin{aligned}
w(u ; n)= & q^{n+u+1}(1-q)^{n-1} \frac{u+1}{n+u+1} \sum_{k=1}^{n}\left(\begin{array}{c}
n+u+1 \\
k
\end{array}\right)\left(\begin{array}{c}
n-1 \\
n-k
\end{array}\right) \\
& -q^{n+u+1}(1-q)^{n-1} \frac{u}{n+u} \sum_{k=1}^{n}\left(\begin{array}{c}
n+u \\
k
\end{array}\right)\left(\begin{array}{c}
n-1 \\
n-k
\end{array}\right) \\
= & q^{n+u+1}(1-q)^{n-1}\left[\frac{u+1}{n+u+1}\left(\begin{array}{c}
2 n+u \\
n
\end{array}\right)-\frac{u}{n+u}\left(\begin{array}{c}
2 n+u-1 \\
n
\end{array}\right)\right] \\
= & q^{n+u+1}(1-q)^{n-1} \frac{u+2}{2 n+u}\left(\begin{array}{c}
2 n+u \\
n-1
\end{array}\right), \quad n \in \mathbb{N}^{+} .
\end{aligned}
$$

Remark 4.1. When $u=0$, we have

$$
w(0 ; n)=\frac{\theta}{1-\theta} \frac{1}{n+1} \sum_{k=1}^{n}\left(\begin{array}{c}
n+1 \\
k
\end{array}\right)\left(\begin{array}{c}
n-1 \\
n-k
\end{array}\right)[q(1-\theta)]^{k}[\theta(1-q)]^{n-k}, \quad n \in \mathbb{N}^{+} \text {. }
$$

In particular, if $\theta=q$,

$$
w(0 ; n)=q^{n+1}(1-q)^{n-1} \frac{\left(\begin{array}{c}
2 n \\
n
\end{array}\right)}{n+1}, \quad n \in \mathbb{N}^{+}
$$

Example 4.2. (Constant claims of size 2) If $X_{i} \equiv 2$ for $i \in \mathbb{N}^{+}$, i.e., $p(x)=I(x=2)$, then $b(0)=1-q$ and $b(2)=q$. It follows from Li $(2005 b), p .259$, that

$$
\phi_{T}(u)=\frac{1}{R^{u+1}}, \quad u \in \mathbb{N},
$$


where $R>1$ is the solution to the simplified Lundberg's equation

$$
s=v \hat{b}(s)=v\left(1-q+q s^{2}\right)
$$

Solving the equation above gives

$$
R=\frac{1+\sqrt{1-4(1-q) q v^{2}}}{2 q v}, \quad \rho=\frac{1-\sqrt{1-4(1-q) q v^{2}}}{2 q v},
$$

so that multiplying the latter equalities yields $R=(1-q) /(q \rho)$ and

$$
\phi_{T}(u)=\left(\frac{1-q}{q}\right)^{u+1} \rho^{u+1}
$$

Substituting (2.4) into (4.10) yields

$$
\phi_{T}(u)=(u+1)\left(\frac{1-q}{q}\right)^{u+1} \sum_{n=u+1}^{\infty} \frac{v^{n}}{n} b^{n *}(n-u-1) .
$$

Subsequent inversion results in

$$
w(u ; n)= \begin{cases}0, & n \leq u \\ (u+1)\left(\frac{1-q}{q}\right)^{u+1} \frac{b^{n *}(n-u-1)}{n}, & n>u\end{cases}
$$

To find $b^{n *}(x)$, we note that

$$
\sum_{k=0}^{\infty} s^{k} b^{n *}(k)=[\hat{b}(s)]^{n}=\left(1-q+q s^{2}\right)^{n}=\sum_{k=0}^{n}\left(\begin{array}{l}
n \\
k
\end{array}\right)(1-q)^{n-k} q^{k} s^{2 k} .
$$

Equating the coefficients gives for $x=0,1, \ldots, 2 n$,

$$
b^{n *}(x)=\left\{\begin{array}{ll}
0, & x=2 k+1 \\
\left(\begin{array}{l}
n \\
k
\end{array}\right)(1-q)^{n-k} q^{k}, & x=2 k
\end{array} .\right.
$$

We provide the general expression for $w(u ; m)$ in the following theorem.

Theorem 4.1. Given an initial surplus of $u>0$, the time of ruin $T$ has $p . f$.

$$
w(u ; m)=\mathbb{P}\{T=m \mid U(0)=u\}=\frac{1}{m} \sum_{x=1}^{m} x \zeta(u, x) b^{m *}(m-x), \quad u, m \in \mathbb{N}^{+},
$$


where

$$
\zeta(u, x)=\frac{q \mu_{1}}{1-q} b_{1}(x)+\sum_{n=1}^{u}\left[\frac{q \mu_{1}}{1-q} \sum_{y=1}^{x} b_{1}(x-y) \sigma_{n}(u, y)-\sigma_{n}(u, x)\right]
$$

and

$$
\sigma_{n}(u, y)=\left(\frac{q}{1-q}\right)^{n} \sum_{j=0}^{n-1}(-1)^{j}\left(\begin{array}{c}
n \\
j
\end{array}\right) \sum_{x=0}^{u-n}\left(\begin{array}{c}
x+n-1 \\
n-1
\end{array}\right) P^{j *}(u-n+j-x) p^{(n-j) *}(y+n-j+x)
$$

for $u \geq n \in \mathbb{N}^{+}, y \in \mathbb{N}^{+}$.

Proof. It follows from Theorem 5 in Li and Garrido (2002) that $\phi_{T}(u)=E\left\{v^{T} I(T<\infty) \mid U(0)=u\right\}$ has the compound geometric distribution

$$
\phi_{T}(u)=\sum_{n=1}^{\infty}\left[1-\phi_{T}(0)\right] \overline{G^{n *}}(u), \quad u \in \mathbb{N},
$$

where $G(u)=\sum_{y=1}^{u} g(y \mid 0), u \in \mathbb{N}^{+}$, is the defective c.d.f. of the deficit at ruin with no initial surplus. Observe that if $f_{X Y T}(x, y, t \mid u), x, u \in \mathbb{N}, y, t \in \mathbb{N}^{+}$is the joint (defective) p.f. of the surplus prior to ruin, $x$, the deficit at ruin $y$, and the time of ruin, $t$, starting with an initial surplus of $u$, then

$$
\phi_{T}(u)=\sum_{x=0}^{\infty} \sum_{y=1}^{\infty} \sum_{t=1}^{\infty} v^{t} f_{X Y T}(x, y, t \mid u) .
$$

Therefore, identity (4.2) implies that the (defective) zero-truncated p.f. of the deficit at ruin is

$$
\begin{aligned}
g(y \mid 0) & =\sum_{x=0}^{\infty} \sum_{t=1}^{\infty} v^{t} f_{X Y T}(x, y, t \mid 0) \\
& =\frac{1}{b(0)} \sum_{x=0}^{\infty} \rho^{x+1} b(x+y+1), \quad y \in \mathbb{N}^{+} .
\end{aligned}
$$

Also, $\overline{G^{n *}}(u)=\left[\phi_{T}(0)\right]^{n}-G^{n *}(u)$ is the (defective) tail distribution of the $n$-fold convolution of $g(\cdot \mid 0)$ with itself.

It is more convenient to express $\phi_{T}(u)$ as

$$
\begin{aligned}
\phi_{T}(u) & =\phi_{T}(0)+\sum_{n=1}^{\infty} \phi_{T}(0) G^{n *}(u)-\sum_{n=1}^{\infty} G^{n *}(u) \\
& =\phi_{T}(0)+\sum_{n=1}^{u} \phi_{T}(0) G^{n *}(u)-\sum_{n=1}^{u} G^{n *}(u), \quad u \in \mathbb{N}^{+},
\end{aligned}
$$

as $G^{n *}(u)=0$ if $n>u$. 
To invert $G^{n *}(u)=\sum_{y=n}^{u} g^{n *}(y \mid 0)$ with respect to $\rho$, we note that $\hat{g}^{n *}(z):=\sum_{y=n}^{\infty} z^{y} g^{n *}(y \mid 0)=$ $\hat{g}^{n}(z)$ with

$$
\begin{aligned}
\hat{g}(z) & =\sum_{y=1}^{\infty} z^{y} g(y \mid 0)=\frac{1}{b(0)} \sum_{y=1}^{\infty} z^{y} \sum_{x=0}^{\infty} \rho^{x+1} b(x+y+1) \\
& =\frac{1}{b(0)} \frac{(z / \rho) \sum_{x=1}^{\infty} \rho^{x} b(x)-\sum_{x=1}^{\infty} z^{x} b(x)}{1-z / \rho}=\frac{q}{1-q} \frac{(z / \rho) \hat{p}(\rho)-\hat{p}(z)}{1-z / \rho}
\end{aligned}
$$

by the definition (1.4) of $b$. Then,

$$
\begin{aligned}
\hat{g}^{n *}(z) & =\left(\frac{q}{1-q}\right)^{n}\left[\frac{(z / \rho) \hat{p}(\rho)-\hat{p}(z)}{1-z / \rho}\right]^{n} \\
& =\left[\frac{q}{(1-q)(1-z / \rho)}\right]^{n} \sum_{j=0}^{n}(-1)^{j}\left(\begin{array}{c}
n \\
j
\end{array}\right) \hat{p}^{j}(z)\left(\frac{z}{\rho}\right)^{n-j} \hat{p}^{n-j}(\rho) .
\end{aligned}
$$

Let the function $a_{n}(y, k), y \geq n$, be such that $g^{n *}(y \mid 0)=\sum_{k=0}^{\infty} \rho^{k} a_{n}(y, k)$. Then,

$$
\hat{g}^{n *}(z)=[\hat{g}(z)]^{n}=\sum_{y=n}^{\infty} z^{y} \sum_{k=0}^{\infty} \rho^{k} a_{n}(y, k)=\sum_{k=0}^{\infty} \rho^{k} \sum_{y=n}^{\infty} z^{y} a_{n}(y, k) .
$$

It follows from (4.15) that $\hat{g}^{n *}(z)=0$ if $\rho=0$, which implies that

$$
\begin{aligned}
\hat{g}^{n *}(z) & =\sum_{k=1}^{\infty} \rho^{k} \sum_{y=n}^{\infty} z^{y} a_{n}(y, k), \\
g^{n *}(y \mid 0) & =\sum_{k=1}^{\infty} \rho^{k} a_{n}(y, k), \\
G^{n *}(u) & =\sum_{y=n}^{u} g^{n *}(y)=\sum_{k=1}^{\infty} \rho^{k} \sum_{y=n}^{u} a_{n}(y, k)=\sum_{k=1}^{\infty} \rho^{k} \sigma_{n}(u, k), \quad u \geq n,
\end{aligned}
$$

where $\sigma_{n}(u, k)=\sum_{y=n}^{u} a_{n}(y, k)$.

In what follows, we focus on finding $\sigma_{n}(u, k)$. By Graham et al. (1994),

$$
\frac{1}{(1-z / \rho)^{n}}=\sum_{k=0}^{\infty}\left(\begin{array}{c}
k+n-1 \\
n-1
\end{array}\right)\left(\frac{z}{\rho}\right)^{k}
$$

This equation implies that $\hat{p}^{j}(z) /(1-z / \rho)^{n}, j \in \mathbb{N}^{+}$, is the generating function of

$$
\sum_{x=0}^{y-j}\left(\begin{array}{c}
x+n-1 \\
n-1
\end{array}\right) p^{j *}(y-x) \rho^{-x}, \quad y \geq j
$$


Hence, $\hat{p}^{j}(z) z^{n-j} /(1-z / \rho)^{n}, n \geq j \in \mathbb{N}$, is the generating function of

$$
\left\{\begin{array}{ll}
\left(\begin{array}{c}
y-1 \\
n-1
\end{array}\right) \rho^{n-y}, & y \geq n, j=0 \\
\sum_{x=0}^{y-n}\left(\begin{array}{c}
x+n-1 \\
n-1
\end{array}\right) p^{j *}(y-n+j-x) \rho^{-x}, & y \geq n, j \in \mathbb{N}^{+}
\end{array} .\right.
$$

Consequently,

$$
\begin{aligned}
g^{n *}(y \mid 0)= & \left(\frac{q}{1-q}\right)^{n} \sum_{j=1}^{n}(-1)^{j}\left(\begin{array}{l}
n \\
j
\end{array}\right)\left[\frac{\hat{p}(\rho)}{\rho}\right]^{n-j} \sum_{x=0}^{y-n}\left(\begin{array}{c}
x+n-1 \\
n-1
\end{array}\right) p^{j *}(y-n+j-x) \rho^{-x} \\
& +\left(\frac{q}{1-q}\right)^{n}\left[\frac{\hat{p}(\rho)}{\rho}\right]^{n}\left(\begin{array}{c}
y-1 \\
n-1
\end{array}\right) \rho^{n-y}
\end{aligned}
$$

with the convention that a sum with a higher lower limit than upper limit equals zero. Therefore,

$$
\begin{aligned}
G^{n *}(u)= & \sum_{y=n}^{u} g^{n *}(y \mid 0)=\left(\frac{q}{1-q}\right)^{n} \sum_{j=1}^{n}(-1)^{j}\left(\begin{array}{c}
n \\
j
\end{array}\right)\left[\frac{\hat{p}(\rho)}{\rho}\right]^{n-j} \sum_{y=n}^{u} \sum_{x=0}^{y-n}\left(\begin{array}{c}
x+n-1 \\
n-1
\end{array}\right) p^{j *}(y-n+j-x) \rho^{-x} \\
& +\left(\frac{q}{1-q}\right)^{n}\left[\frac{\hat{p}(\rho)}{\rho}\right]^{n} \sum_{x=0}^{u-n}\left(\begin{array}{c}
x+n-1 \\
n-1
\end{array}\right) \rho^{-x} \\
= & \left(\frac{q}{1-q}\right)^{n} \sum_{j=1}^{n}(-1)^{j}\left(\begin{array}{c}
n \\
j
\end{array}\right)\left[\frac{\hat{p}(\rho)}{\rho}\right]^{n-j} \sum_{x=0}^{u-n}\left(\begin{array}{c}
x+n-1 \\
n-1
\end{array}\right) \rho^{-x} \sum_{y=x+n}^{u} p^{j *}(y-n+j-x) \\
& +\left(\frac{q}{1-q}\right)^{n}\left[\frac{\hat{p}(\rho)}{\rho}\right]^{n} \sum_{x=0}^{u-n}\left(\begin{array}{c}
x+n-1 \\
n-1
\end{array}\right) \rho^{-x} \\
= & \left(\frac{q}{1-q}\right)^{n} \sum_{j=1}^{n}(-1)^{j}\left(\begin{array}{c}
n \\
j
\end{array}\right)\left[\frac{\hat{p}(\rho)}{\rho}\right]^{n-j} \sum_{x=0}^{u-n}\left(\begin{array}{c}
x+n-1 \\
n-1
\end{array}\right) P^{j *}(u-n+j-x) \rho^{-x} \\
& +\left(\frac{q}{1-q}\right)^{n}\left[\frac{\hat{p}(\rho)}{\rho}\right]^{n} \sum_{x=0}^{u-n}\left(\begin{array}{c}
x+n-1 \\
n-1
\end{array}\right) \rho^{-x} \\
= & \left(\frac{q}{1-q}\right)^{n} \sum_{j=0}^{n}(-1)^{j}\left(\begin{array}{c}
n \\
j
\end{array}\right)\left[\frac{\hat{p}(\rho)}{\rho}\right]^{n-j} \sum_{x=0}^{u-n}\left(\begin{array}{c}
x+n-1 \\
n-1
\end{array}\right) P^{j *}(u-n+j-x) \rho^{-x}
\end{aligned}
$$

since $P^{0 *}(y)=1, y \geq 0$. Further, observe that for $0 \leq j<n$,

$$
\hat{p}^{n-j}(\rho) \rho^{u-n+j-x}=\sum_{k=n-j}^{\infty} p^{(n-j) *}(k) \rho^{u-n+j-x+k}=\sum_{k=u-x}^{\infty} p^{(n-j) *}(k-u+n-j+x) \rho^{k}
$$

after a change of variables. Thus,

$\rho^{u} G^{n *}(u)=$ 


$$
\begin{aligned}
& \left(\frac{q}{1-q}\right)^{n} \sum_{j=0}^{n-1}(-1)^{j}\left(\begin{array}{c}
n \\
j
\end{array}\right) \sum_{x=0}^{u-n}\left(\begin{array}{c}
x+n-1 \\
n-1
\end{array}\right) P^{j *}(u-n+j-x) \sum_{k=u-x}^{\infty} p^{(n-j) *}(k-u+n-j+x) \rho^{k} \\
& +\left(\frac{-q}{1-q}\right) \sum_{x=0}^{n-n}\left(\begin{array}{c}
x+n-1 \\
n-1
\end{array}\right) P^{n *}(u-x) \rho^{u-x} \\
& =\left(\frac{q}{1-q}\right) \sum_{j=0}^{n-1}(-1)^{j}\left(\begin{array}{c}
n \\
j
\end{array}\right) \sum_{k=n}^{u}\left[\sum_{x=u-k}^{u-n}\left(\begin{array}{c}
x+n-1 \\
n-1
\end{array}\right) P^{j *}(u-n+j-x) p^{(n-j) *}(k-u+n-j+x)\right] \rho^{k} \\
& +\left(\frac{q}{1-q}\right)^{n} \sum_{j=0}^{n-1}(-1)^{j}\left(\begin{array}{c}
n \\
j
\end{array}\right) \sum_{k=u+1}^{\infty}\left[\sum_{x=0}^{u-n}\left(\begin{array}{c}
x+n-1 \\
n-1
\end{array}\right) P^{j *}(u-n+j-x) p^{(n-j) *}(k-u+n-j+x)\right] \rho^{k} \\
& +\left(\frac{-q}{1-q}\right)^{n} \sum_{k=n}^{u}\left(\begin{array}{c}
u-k+n-1 \\
n-1
\end{array}\right) P^{n *}(k) \rho^{k}
\end{aligned}
$$

by exchanging the order of summation.

As

$$
\rho^{u} G^{n *}(u)=\sum_{k=1}^{\infty} \sigma_{n}(u, k) \rho^{u+k}=\sum_{k=u+1}^{\infty} \sigma_{n}(u, k-u) \rho^{k},
$$

the above derivations imply that equating the coefficients of $\rho^{k}$ for $k \geq u+1$ produces

$\sigma_{n}(u, k-u)=\left(\frac{q}{1-q}\right)^{n} \sum_{j=0}^{n-1}(-1)^{j}\left(\begin{array}{c}n \\ j\end{array}\right) \sum_{x=0}^{u-n}\left(\begin{array}{c}x+n-1 \\ n-1\end{array}\right) P^{j *}(u-n+j-x) p^{(n-j) *}(k-u+n-j+x)$,

or equivalently,

$$
\sigma_{n}(u, y)=\left(\frac{q}{1-q}\right)^{n} \sum_{j=0}^{n-1}(-1)^{j}\left(\begin{array}{c}
n \\
j
\end{array}\right) \sum_{x=0}^{u-n}\left(\begin{array}{c}
x+n-1 \\
n-1
\end{array}\right) P^{j *}(u-n+j-x) p^{(n-j) *}(y+n-j+x),
$$

for $u \geq n \in \mathbb{N}^{+}, y \in \mathbb{N}^{+}$. Finally, combining equations (4.14) and (4.4) yields

$$
\phi_{T}(u)=\sum_{y=1}^{\infty} \zeta(u, y) \rho^{y}=\sum_{n=1}^{\infty} w(u ; n) v^{n}
$$

with $\zeta$ defined by (4.12). Finally, relationship (2.2) together with expression (2.3) yield the required identity (4.11)

Remark 4.2. The explicit expression for $w(u ; n)$ in $(4.11)$ is computationally tractable and readily programmable as $w(u ; n), \zeta(u, x)$ and $\sigma_{k}(u, y)$ are expressed in summations of finite terms. Moreover, $b^{n *}(x)$ and $p^{k *}(x)$ may be evaluated recursively as in (2.5) for any discrete distributions of $b$ and $p$. In particular, closed-form formulas exist for $b^{n *}(x), p^{k *}(x)$, and $P^{k *}(x)$ when claims follow a discrete phase-type distribution. 
Dickson (2007) uses probabilistic arguments to derive the density of the time of ruin in the classical risk model. We now apply the same argument to the compound binomial risk model to give a simpler explicit expression for $w(u ; n), u \in \mathbb{N}$ :

$$
\begin{aligned}
w(u ; 1)= & q \bar{P}(u+1), \quad u \in \mathbb{N}, \\
w(u ; n)= & q \sum_{x=1}^{n+u} b^{(n-1) *}(n+u-x) \bar{P}(x) \\
& -(1-q) \sum_{m=1}^{n-1} w(0 ; n-m) b^{(m-1) *}(m+u), \quad n>1,
\end{aligned}
$$

where $w(0 ; n)$ is calculated using $(4.1)$ or $(4.5)$.

The first term in (4.16) represents the probability that the total amount of claims by time $n-1$ is $u+n-x$, so that the surplus at time $n-1$ is $x, 1 \leq x \leq n+u$, and a claim of size greater than $x$ occurs at time $n$ with a probability $q$. The first term makes allowance for the possibility that the surplus was below zero prior to time $n$ and its probability is represented by the second term. Suppose that at time $m-1 \leq n-2$ the aggregate claim amount is $u+m$, so that the surplus at time $m-1$ is -1 and the surplus at time $m$ is 0 if there is no claim in $(m-1, m]$ with probability $(1-q)$. The probability that the surplus will be negative at time $n$ from time $m$ is $w(0 ; n-m)$, meaning that the surplus falls below zero at time $n$. Summing over $x$ and $m$ gives the adjustment to the probability in the first term of equation (4.16).

For the discrete-time risk model, $w(u ; n)$ may also be evaluated recursively, see Dickson et al. (1995) or Dickson (2005). In the following two examples, we will compare the time of numerical computation in calculating the probability function of the time of ruin using the two explicit formulae and the recursive formula for $w(u ; n)$.

Example 4.3. In this example, we assume that $p(x)=(1-\theta)^{2} x \theta^{x-1}, x \in \mathbb{N}^{+}, \theta \in(0,1)$, so that $b(0)=1-q$ and $b(x)=q p(x), x \in \mathbb{N}^{+}$. We set $q=0.2$ and $\theta=0.6$ so that $q \mu_{1}=0.8<1$. Table 1 (left-hand side) gives $w(u ; n)$ for $u=0,5,10,15$ and $n=1,2, \ldots, 15$ using the recursive formula in Dickson (2005) and the explicit formulas (4.11) and (4.16). The three methods give the same values as formulas involved in the three methods are of finite summations and no truncation is needed. The formulas for $w(u ; n)$ in all three methods are programmed using $\mathrm{C}++2010$ Express installed in a computer with Intel core i7 CPU. In computing $w(3 ; 3)$, the recursive formula in Dickson (2005) needs $1.6 \times 10^{-5}$ seconds, formula (4.16) needs $3.1 \times 10^{-5}$ seconds, while formula (4.11) needs 0.002 seconds. When computing $w(10 ; 10)$, recursive formula in Dickson (2005) needs 1.584 seconds, formula (4.16) needs 0.298 seconds, while formula (4.11) needs 5.646 seconds. When $u=6, n=6$, formula (4.16) is much faster than the recursive formula, while the recursive formula is faster than formula (4.11). 


\begin{tabular}{|c|c|c|c|c|c|c|c|c|}
\hline \multicolumn{7}{|c|}{$w(u ; n)$ for Example 4.3} & \multicolumn{5}{c|}{$w(u ; n)$ for Example 4.4} \\
\hline$n$ & $u=0$ & $u=5$ & $u=10$ & $u=15$ & $u=0$ & $u=5$ & $u=10$ & $u=15$ \\
\hline 1 & 0.16800 & 0.03173 & 0.00392 & 0.00042 & 0.03803 & 0.00055 & 0.000101 & 0.0000342 \\
\hline 2 & 0.10906 & 0.02998 & 0.00621 & 0.00083 & 0.02215 & 0.00050 & 0.000094 & 0.0000180 \\
\hline 3 & 0.07279 & 0.02745 & 0.00747 & 0.00102 & 0.01376 & 0.00045 & 0.000087 & 0.0000103 \\
\hline 4 & 0.05125 & 0.02476 & 0.00812 & 0.00112 & 0.00903 & 0.00039 & 0.000080 & 0.0000063 \\
\hline 5 & 0.03812 & 0.02224 & 0.00839 & 0.00116 & 0.00620 & 0.00035 & 0.000074 & 0.0000041 \\
\hline 6 & 0.02969 & 0.01998 & 0.00844 & 0.00115 & 0.00442 & 0.00031 & 0.000068 & 0.0000029 \\
\hline 7 & 0.02395 & 0.01802 & 0.00828 & 0.00112 & 0.00325 & 0.00027 & 0.000063 & 0.0000020 \\
\hline 8 & 0.01984 & 0.01632 & 0.00796 & 0.00108 & 0.00245 & 0.00024 & 0.000057 & 0.0000015 \\
\hline 9 & 0.01677 & 0.01484 & 0.00758 & 0.00103 & 0.00189 & 0.00021 & 0.000050 & 0.0000011 \\
\hline 10 & 0.01440 & 0.01355 & 0.00716 & 0.00097 & 0.00148 & 0.00019 & 0.000044 & 0.0000009 \\
\hline 11 & 0.01254 & 0.01243 & 0.00672 & 0.00091 & 0.00118 & 0.00017 & 0.000037 & 0.0000007 \\
\hline 12 & 0.01103 & 0.01142 & 0.00628 & 0.00085 & 0.00095 & 0.00015 & 0.000032 & 0.0000005 \\
\hline 13 & 0.00980 & 0.01050 & 0.00586 & 0.00079 & 0.00077 & 0.00014 & 0.000026 & 0.0000004 \\
\hline 14 & 0.00878 & 0.00967 & 0.00545 & 0.00073 & 0.00064 & 0.00012 & 0.000022 & 0.0000004 \\
\hline 15 & 0.00792 & 0.00891 & 0.00506 & 0.00068 & 0.00053 & 0.00011 & 0.000018 & 0.0000003 \\
\hline
\end{tabular}

Table 1: $w(u ; n)$ for Examples 4.3 and 4.4

Example 4.4. In this example, we assume that claim sizes follow a zeta distribution (also called a discrete Pareto) with

$$
p(x)=\frac{1}{\zeta(a) x^{a}}, \quad a>1, x \in \mathbb{N}^{+},
$$

where

$$
\zeta(a)=\sum_{n=1}^{\infty} \frac{1}{n^{a}}, \quad a>1,
$$

is the Riemann zeta function. We assume that $q=0.5$ and $a=4$ so that $\mu_{1}=\zeta(3) / \zeta(4)=1.11065$. Table 1 (right-hand side) provides the values of $w(u ; n)$ for $u=0,5,10,15$ and $n=1,2, \ldots, 15$. As the expected claim size is very small, $w(u ; n)$ are significantly small. Similarly to our findings in Example 4.3, the recursive formula is faster than the other explicit formulas when $n$ and $u$ are small, while Formula (4.16) is much faster than the other two formulas when $n$ and $u$ become large. 


\section{The probability function of the duration of the negative surplus}

In this section, we study the probability function of the duration of the negative surplus. For the surplus process $U(n)$, once ruin has occurred at time $T$, the process will cross again the level zero at time $T+\bar{T}_{1}$ for the first time, where $\bar{T}_{1}$ is the duration of the first negative surplus. Let $\bar{T}_{j}, j=2,3, \ldots$, be the length of time while the surplus is negative for the $j$ th time. Then $\bar{T}_{2}, \bar{T}_{3}, \ldots$, are i.i.d. and are independent of $\bar{T}_{1}$. We define $M$ to be the number of occasions on which the surplus process falls below zero and

$$
\bar{T}=\sum_{i=1}^{M} \bar{T}_{i}, \quad(=0, \text { if } M=0)
$$

to be the total duration of negative surplus. Following the same line of reasoning as in Dos Reis (1993), we conclude that $M$ has a zero-modified geometric distribution with p.f.

$$
\mathbb{P}\{M=n\}=\left\{\begin{array}{ll}
1-\psi(u), & n=0 \\
{[1-\psi(0)][\psi(0)]^{n-1} \psi(u),} & n \in \mathbb{N}^{+}
\end{array} .\right.
$$

Let $d_{u}(n)=\mathbb{P}\left\{\bar{T}_{1}=n \mid T<\infty, U(0)=u\right\}$ be the probability function of the duration of the first negative surplus, given that ruin occurs starting from an initial surplus of $u$. Then $d_{0}(n)$ is the p.f. of $\bar{T}_{2}, \bar{T}_{3}, \ldots$ Define for $v \in(0,1)$

$$
\xi_{v}(u)=E\left\{v^{\bar{T}_{1}} \mid T<\infty, U(0)=u\right\}, \quad u \in \mathbb{N},
$$

to be the p.g.f. of $\bar{T}_{1}$. Clearly, $\xi_{v}(0)$ is the p.g.f. of $\bar{T}_{j}$ for $j=2,3, \ldots$

Proposition 5.1. The probability function $d_{u}$ of the duration of the negative surplus $\bar{T}_{1}$ satisfies

$$
d_{u}(n)=\frac{1}{n} \sum_{y=1}^{n} y \bar{g}(y \mid u) b^{n *}(n-y), \quad u \in \mathbb{N}, n \in \mathbb{N}^{+},
$$

where $\bar{g}(y \mid u)=g(y \mid u) / \psi(u)$ is the proper p.f. of the deficit at ruin, given that ruin occurs from an initial surplus level of $u$.

Proof. On one hand, by definition,

$$
\xi_{v}(u)=\sum_{n=1}^{\infty} v^{n} d_{u}(n) .
$$

On the other hand, after the surplus drops to a level $-y<0$, the time until it reaches zero again has the same distribution as time $T_{y}$ defined by (3.1). Hence, the Total Probability Theorem implies that

$$
\xi_{v}(u)=\sum_{y=1}^{\infty} \mathbb{E}\left\{v^{T_{y}}\right\} \bar{g}(y \mid u)=\sum_{y=1}^{\infty} \rho^{y} \bar{g}(y \mid u) .
$$


The desired result follows immediately by Lemma 2.1 .

Recall that (see also Lemma 1.1) when $v \rightarrow 1$, then $\rho \rightarrow 1$. Consequently, identity (4.13) yields $g(y \mid 0)=q \mu_{1} b_{1}(y) /(1-q)$. By implementing equation (5.1), the above proposition yields the following simple result.

Corollary 5.1.1. When $u=0$, we have

$$
d_{0}(n)=\frac{w(0 ; n)}{\psi(0)}, \quad n \in \mathbb{N}^{+} .
$$

We may interpret Corollary 5.1.1 in the following way: When $u=0, \bar{T}_{1}$ and $T \mid T<\infty$ have the same distribution. We have the discrete analogue of the corresponding result under the compound Poisson risk model (see Egídio dos Reis, 1993).

Corollary 5.1.2. (Geometric claims) If $p(x)=(1-\theta) \theta^{x-1}, x \in \mathbb{N}^{+}, \theta \in(0,1)$, then $\bar{g}(y \mid u)=\bar{g}(y \mid 0)$ due to the memoryless property of the geometric distribution. Consequently, $\bar{T}_{1}$ and $\bar{T}_{j}, j=2,3, \ldots$, have the same distribution, i.e., for $u \in \mathbb{N}^{+}$,

$$
d_{u}(n)=d_{0}(n)=\frac{w(0 ; n)}{\psi(0)}, \quad n \in \mathbb{N}^{+},
$$

where $w(0 ; n)$ is given in (4.9).

Proposition 5.2. The probability function $d_{u}$ of the duration of the negative surplus may be calculated recursively by

$$
\begin{aligned}
d_{u}(n) & =\frac{q \mu_{1}}{(1-q)}\left[\sum_{x=1}^{u} d_{u-x}(n) b_{1}(x)+\frac{1}{n \psi(u)} \sum_{y=1}^{n} y b_{1}(u+y) b^{n *}(n-y)\right] \\
& =\frac{q \mu_{1}}{(1-q)}\left[\sum_{x=1}^{u} d_{u-x}(n) b_{1}(x)+\frac{1}{\psi(u)} \sum_{y=1}^{n} b_{1}(u+y) f(y, n)\right]
\end{aligned}
$$

where the starting value is $d_{0}(n)=w(0 ; n) / \psi(0)$.

Proof. It follows from Li and Garrido (2002) that $\bar{g}(y \mid u)$ may be calculated recursively by

$$
\bar{g}(y \mid u)=\frac{q \mu_{1}}{(1-q)}\left[\sum_{x=1}^{u} \bar{g}(y \mid u-x) b_{1}(x)+\frac{b_{1}(u+y)}{\psi(u)}\right], \quad u, y \in \mathbb{N}^{+} .
$$

Substituting the formula above into (5.1) yields the recursive for $d_{u}(n)$ in (5.2). 
The probability function of the total duration of negative surplus $\bar{T}=\sum_{i=1}^{M} \bar{T}_{i}$ may be calculated recursively as the distribution of $M$ belongs to the $(a, b, 1)$ class, see Sundt and Jewell (1981). In particular, if claim amounts follow a geometric distribution, i.e., $p(x)=(1-\theta) \theta^{x-1}, x \in \mathbb{N}^{+}, \theta \in(0,1)$, then the probability function of $\bar{T}$ has the following formula:

$$
\begin{aligned}
\bar{d}_{u}(0) & =\mathbb{P}\{\bar{T}=0\}=\mathbb{P}\{M=0\}=\phi(u) \\
\bar{d}_{u}(n) & =\mathbb{P}\{\bar{T}=n\}=\psi(u) \phi(0) \sum_{k=0}^{\infty}[\psi(0)]^{k} d_{0}^{(k+1) *}(n) \\
& =\frac{\psi(u) \phi(0)}{\psi(0)} \sum_{k=1}^{\infty} w^{k *}(0 ; n)=\frac{\psi(u) \phi(0)}{\psi(0)} \sum_{k=1}^{n} w^{k *}(0 ; n), \quad n=1,2, \ldots
\end{aligned}
$$

where $w(0 ; n)$ is given by $(4.9)$ and $\psi(u)$ is given in (4.8).

We will show that when $q=\theta$, there is a simple formula for $\sum_{k=1}^{n} w^{k *}(0 ; n)$. Note that

$$
\begin{aligned}
\hat{w}(0 ; z) & =\sum_{n=1}^{\infty} z^{n} w(0 ; n)=\sum_{n=1}^{\infty} z^{n}(1-q)^{n-1} q^{n+1} \frac{\left(\begin{array}{c}
2 n \\
n
\end{array}\right)}{n+1} \\
& =\frac{q}{1-q} \sum_{n=1}^{\infty}[(1-q) q z]^{n} \frac{\left(\begin{array}{c}
2 n+1 \\
n
\end{array}\right)}{2 n+1} \\
& =\frac{q}{1-q}\left[\mathcal{B}_{2}((1-q) q z)-1\right]=\frac{q}{1-q}(1-q) q z\left[\mathcal{B}_{2}((1-q) q z)\right]^{2},
\end{aligned}
$$

where

$$
\mathcal{B}_{t}(z)=\sum_{m=0}^{\infty}\left(\begin{array}{c}
t m+1 \\
m
\end{array}\right) \frac{z^{m}}{t m+1}
$$

is the generalized binomial function which has the remarkable property

$$
\left[\mathcal{B}_{t}(z)\right]^{k}=\sum_{m=0}^{\infty}\left(\begin{array}{c}
t m+k \\
m
\end{array}\right) \frac{k}{t m+k} z^{m}
$$

See Graham et al. (1994) for details. Then,

$$
\begin{aligned}
{[\hat{w}(0 ; z)]^{k} } & =q^{2 k} z^{k}\left[\mathcal{B}_{2}((1-q) q z)\right]^{2 k}=q^{2 k} z^{k} \sum_{m=0}^{\infty}\left(\begin{array}{c}
2 m+k \\
m
\end{array}\right) \frac{k}{2 m+k}[(1-q) q z]^{m} \\
& =\left(\frac{q}{1-q}\right)^{k} \sum_{n=k}^{\infty}\left(\begin{array}{c}
2 n-k \\
n-k
\end{array}\right) \frac{k}{2 n-k}[(1-q) q z]^{n}
\end{aligned}
$$

Inverting $[\hat{w}(0 ; z)]^{k}$ produces

$$
w^{k *}(0 ; n)=\left(\frac{q}{1-q}\right)^{k}\left(\begin{array}{c}
2 n-k \\
n-k
\end{array}\right) \frac{k}{2 n-k}[(1-q) q]^{n}, \quad n \geq k .
$$


Substituting it into (5.3) yields for $n \in \mathbb{N}^{+}$,

$$
\begin{aligned}
\bar{d}_{u}(n) & =\frac{\psi(u) \phi(0)}{\psi(0)}[(1-q) q]^{n} \sum_{k=0}^{n}\left(\frac{q}{1-q}\right)^{k}\left(\begin{array}{c}
2 n-k \\
n-k
\end{array}\right) \frac{k}{2 n-k} \\
& =\frac{\psi(u) \phi(0)}{\psi(0)} q^{2 n} \sum_{m=0}^{n}\left(\begin{array}{c}
n+m \\
m
\end{array}\right) \frac{n-m}{n+m}\left(\frac{1-q}{q}\right)^{m} .
\end{aligned}
$$

Example 5.1. Table 2 lists the values for $d_{u}(n)$ when $u=0,5,10,15$ and some values for $n=$ $1,2, \ldots, 30$ where model parameters are set to be the same as those in Examples 4.3 and 4.4. For each example, for a fixed initial surplus, $d_{u}(n)$ is decreasing in $n$; for a fixed $n, d_{u}(n)$ is decreasing in $u$, i.e., the larger the initial surplus, the less time the negative surplus lasts given that ruin has occurred.

\begin{tabular}{|c|c|c|c|c|c|c|c|c|}
\hline & \multicolumn{4}{|c}{$d_{u}(n)$ for Example 4.3} & \multicolumn{3}{c|}{$d_{u}(n)$ for Example 4.4} \\
\hline$n$ & $u=0$ & $u=5$ & $u=10$ & $u=15$ & $u=0$ & $u=5$ & $u=10$ & $u=15$ \\
\hline 1 & 0.23143 & 0.21784 & 0.15497 & 0.10473 & 0.34482 & 0.12032 & 0.08214 & 0.06531 \\
\hline 2 & 0.15023 & 0.13032 & 0.09194 & 0.06203 & 0.20081 & 0.09401 & 0.06968 & 0.05743 \\
\hline 3 & 0.10028 & 0.08304 & 0.05828 & 0.03928 & 0.12476 & 0.07599 & 0.06057 & 0.05158 \\
\hline 4 & 0.07060 & 0.05705 & 0.03993 & 0.02690 & 0.08189 & 0.06292 & 0.05350 & 0.04693 \\
\hline 5 & 0.05252 & 0.04193 & 0.02930 & 0.01973 & 0.05625 & 0.05303 & 0.04779 & 0.04308 \\
\hline 10 & 0.01984 & 0.01555 & 0.01085 & 0.00730 & 0.01341 & 0.02638 & 0.02979 & 0.03004 \\
\hline 15 & 0.01091 & 0.00850 & 0.00592 & 0.00399 & 0.00482 & 0.01518 & 0.02016 & 0.02216 \\
\hline 20 & 0.00704 & 0.00547 & 0.00381 & 0.00257 & 0.00216 & 0.00951 & 0.01431 & 0.01688 \\
\hline 30 & 0.00373 & 0.00289 & 0.00201 & 0.00135 & 0.00064 & 0.00439 & 0.00795 & 0.01046 \\
\hline
\end{tabular}

Table 2: $d_{u}(n)$ for Example 5.1

\section{Acknowledgments}

The authors would like to thank an anonymous referee for constructive comments and suggestions, which lead to the significant improvement of the paper. The authors would also like to thank Mr. Can Jin for his help with the numerical calculations throughout the paper. In this research, support by a grant from the Natural Sciences and Engineering Council of Canada is gratefully acknowledged by Kristina Sendova. 


\section{References}

Cheng, S., Gerber, H.U. and Shiu, E.W. (2000) Discounted probabilities and ruin theory in the compound binomial model. Insurance: Mathematics and Economics 26, 239-250.

Cossette, H., Landriault, D. and E. Marceau (2003) Ruin probabilities in the Compound Markov Binomial Model. Scandinavian Actuarial Journal, 301-323.

Cossette, H., Landriault, D. and E. Marceau (2004a) Compound binomial risk model in a Markovian environment. Insurance: Mathematics and Economics 35, 425-443.

Cossette, H., Landriault, D. and E. Marceau (2004b) Exact expressions and upper bound for ruin probabilities in the compound Markov binomial model. Insurance: Mathematics and Economics 34, 449-466.

Dickson, D.C.M. (1994) Some comments on the compound binomial model. ASTIN Bulletin 24, 33-45.

Dickson, D.C.M. (2005) Insurance risk and ruin. Cambridge University Press.

Dickson, D.C.M. (2007) Some finite time ruin problems. Annals of Actuarial Science 2, 217-232.

Dickson, D.C.M., Egídio dos Reis, A.D. and Waters, H.R. (1995) Some stable algorithms in ruin theory and their applications. ASTIN Bulletin 25, 153-175.

Dickson, D.C.M. and Willmot, G.E. (2005) The density of the time to ruin in the classical Poisson risk model. ASTIN Bulletin 35, 45-60.

Egídio dos Reis, A.D. (1993) How long is the surplus below zero? Insurance: Mathematics and Economics 12, 23-38.

Gerber, H.U. (1988) Mathematical fun with the compound binomial process. ASTIN Bulletin 18, $161-168$.

Goulden, I.P. and Jackson, D.M. (1983) Combinatorial enumeration. John. Wiley \& Sons, Inc., Somerset, New Jersey.

Graham, R.L., Knuth, D.E., and Patashnik, O. (1994) Concrete Mathematics, 2nd edition. AddisonWesley, Upper Saddle River, NJ.

Landriault, D. (2008) On a generalization of the expected discounted penalty function in a discretetime insurance risk model. Applied Stochastic Models in Business and Industry 24(6), 525-539. 
Latouche, G. and Ramaswami, V., (1999) Introduction to matrix analytic methods in stochastic modeling. ASA SIAM, Philadelphia.

Li, S. (2005a) Distributions of the surplus before ruin, the deficit at ruin and the claim causing ruin in a class of discrete time risk models. Scandinavian Actuarial Journal (4), 271-284.

Li, S. (2005b) On a class of discrete time renewal risk models. Scandinavian Actuarial Journal (4), 241-60.

Li, S. and Garrido, J. (2002) On the time value of ruin in the discrete-time risk model. Working Paper 02-18, Universidad Carlos III de Madrid, 28 pages.

Panjer, H.H. (1981) Recursive evaluation of a family of compound distributions. ASTIN Bulletin 12, $22-26$.

Pavlova, K.P. and Willmot, G.E. (2004) The discret stationary renewal model and the Gerber-Shiu function Insurance: Mathematics and Economics 35, 267-277.

Shiu, E.S.W. (1989) The probability of eventual ruin in the compound binomial model. ASTIN Bulletin 19, 179-190.

Sundt, B. and Jewell, W. (1981) Further results on recursive evaluation of compound distributions. ASTIN Bulletin 12, 27-39.

Willmot, G.E. (1993) Ruin probabilities in the compound binomial model. Insurance Mathematics and Economics 12, 133-142.

Yang, H., Zhang, Z. and Lan, C. (2009) Ruin problems in a discrete Markov risk model. Statistics and probability Letters 79(1), 21-28.

Yuen, K.C. and Guo, J.Y. (2001) Ruin probabilities for time-correlated claims in the compound binomial model. Insurance: Mathematics and Economics 29, 47-57.

Yuen, K.C. and Guo, J.Y. (2006) Some results on the compound Markov binomial model. Scandinavian Actuarial Journal (3), 129-140. 


\section{University Library}

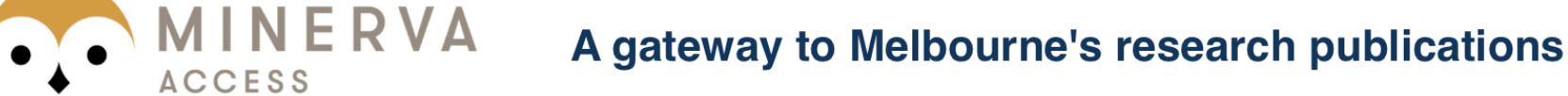

Minerva Access is the Institutional Repository of The University of Melbourne

Author/s:

$\mathrm{Li}, \mathrm{S}$;Sendova, KP

Title:

The finite-time ruin probability under the compound binomial risk model

Date:

2013-07-01

Citation:

Li, S. \& Sendova, K. P. (2013). The finite-time ruin probability under the compound binomial risk model. EUROPEAN ACTUARIAL JOURNAL, 3 (1), pp.249-271. https://doi.org/10.1007/ s13385-013-0063-y.

Persistent Link:

http://hdl.handle.net/11343/282629 